\title{
Operación Cesárea en la Clínica Primero de Mayo
}

\author{
Doctor Carlos Martínez Sáenz \\ Obstetra del ICSS
}

1953

\section{INTRODUCCION}

Quiero presentar este estudio sobre la intervención Cesárea, su incidencia causas y resultados, desde la iniciación de la Clinica Primero de Mayo, hasta el 10 de enero de 1952 fecha en la cual se alcanzó la hospitalización diez mil.

Las fuentes que he consultado son los libros internos de la Clinica y los Archivos del Departamento Médico de ICSS.

Los resultados son parte de la meritoria labor cumplida por los miembros de la Sociedad Colombiana de Obstetricia? Ginecología que colaboran en la nstitución 
Diez mil Hospitalizaciones han dado lugar a un total de 7.909 nacimientos; el resto o sean 2.091 hospitalizaciones corresponden a entradas por abortos falsos trabajos, amenazas de aborto y diversas complicaciones del embarazo.

Hasta el 31 de diciembre del año 1951 se habían efectuado en la Clínica 251 intervenciones cesáreas.

Creo de interés comparar este dato con los que presentan algunas institu. ciones similares, extranjeras ( $\left.{ }^{2}\right)$ y nacionales.

E] Pittsburgh General en 10.499 partos tienen 118 cesáreas.

El Jeffeson Davis de Texas en 22.296 partos, 231 cesáreas. .

El Sloane Hospital de N. Y. en 17.226 partos, 1.000 cesáreas.

El Instituto de Protección Materno Infantil de Bogotá en 10.450 partos tiene 337 cesáreas.

La Clínica de Marly con servicio pensionado únicamente, en 1.675 partos tiene 82 cesáreas.

Algunos porcentajes (1) de hospitales americanos publicados recientemente sirven también como punto de comparación:

New Haven

Margaret Hague

Chicago Lyin-in

5. $9 \%$

2. $6 \%$

New York Hospital ...................... 2. $1 \%$

Boston City Hospital .................... 4. $2 \%$

Kansas City Hospital ..................... 7. 1\%

Johns Hopkins ...................... 5. $5 \%$

Cleveland Maternity .................... 6. $1 \%$

Philadelphia Lvin-in ................. 5. $8 \%$

Sloane Hospital ..................... 5. $8 \%$

Da un promedio igual al $4.9 \%$ en los Americanos ( 1 ).

En Bogotá:

Instituto de Protección Materno-Infantil .......... 3. 18\%

Clínica de Marl . ........................ 4. $8 \%$

Hospital San Jose ..................... 5. $9 \%$

Clínica Primero de Mavo.................. 3. 17\%

El promedio en nuestras Instituciones de $3.4 \%$.

Las 251 cesáreas de la Clinica Primero de Mayo, se reparten en las siguien te forma:

1950 Nacimientos: 2.417 Cesáreas: 94 Porcentaje: $3.8 \%$.

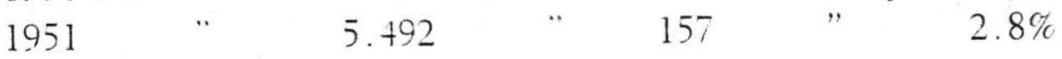

Cabe anotar en estas cifras el hecho de que en el año 51 se presentó un baja del $1 \%$ en la incidencia de cesareas, coincidiendo con las reglamentacione sobre juntas médicas adoptadas. 
Del total de 251 cesáreas, 80 no se pudieron estudiar por diversas causas, o sea $31.8 \%$ no utilizable; el resto, o sean 171 se descomponen en la siguiente forma:

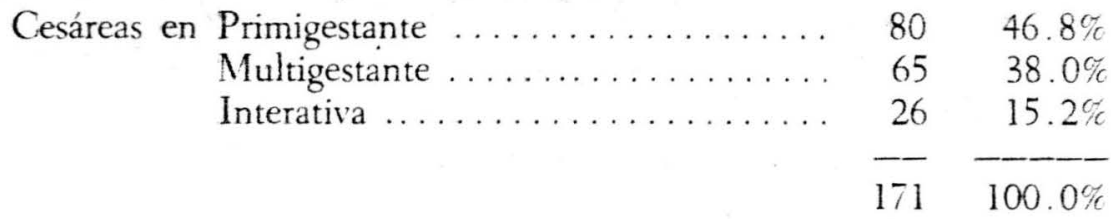

\section{TÉCNICA OPERATORIA}

La técnica operatoria empleada en estas intervenciones fue la segmentaria transperitoneal y no se tuvo en cuenta si se trataba de casos puros o impuros; en este sentido, nuestras cifras se pueden repartir en las siguiente forma:

Casos puros

$110 \quad 64.3 \%$

Impuros

$61 \quad 35.7 \%$

$171 \quad 100.0 \%$

Anestesia:

Se empleó en 154 casos la general Ciclo - Oxígeno - Eter.

$$
\begin{array}{rrr}
16, ", & , & \text { Raquídea. } \\
1 & , \quad, \quad & \text { Pentotal Sódico. }
\end{array}
$$

\section{INDICACIONES}

Las indicaciones apara llevar a efecto estas intervenciones han sido:

Desp. Céfalo-pélvica

Placenta previa

$78 \quad 45.6 \%$

Eclampsia

$2212.8 \%$

Situación Transversa

$21 \quad 12.2 \%$

Desprendimiento de la Placenta

$105.8 \%$

Pelvis infundibular

$52.9 \%$

Distocia cervical

Inercia Uterina

1. $7 \%$

Primigestante de edad avanzada

6. $4 \%$

Deflexión de Cabeza . . . ..................

2. $3 \%$

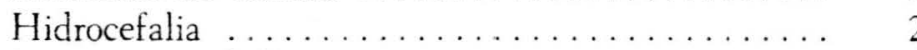

Presentación de Frente . . . . . . . . . . . . . . .

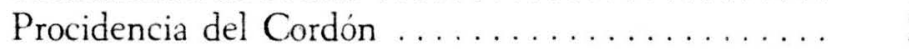

Presentación de Cara ..................

Embarazo gemelar ....................

Esterilidad conyugal prolongada

$4 \quad 2.3 \%$

$21.16 \%$

$21.16 \%$

$21.16 \%$

$2 \quad 1.16 \%$

$10.58 \%$

$1 \quad 0.58 \%$

$1 \quad 0.58 \%$ 
Endocarditis reumatoide

Fístula recto-vaginal

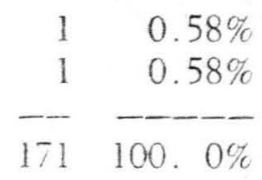

EDAD:

De 15 a 20 años

" 20 a 30

" 30 a 40

" 40 a más

Consideramos sin objeto hacer diferenciación alguna en lo referente a raz Promedio de horas de trabajo .............. 1th. 46.

Promedio de hospitalizaciones

11 dias 8 ho

EDAD DEL EMBBAPAZO:

Cesáreas con feto a término

Las cesáreas con feto prematuro se dividen de la siguiente manera:

Multiparas: 13 .

Primiparas: 7 .

El peso promedio de los niños nacidos por cesarea es de 2.985 gmos. La talla promedio es de $48.5 \mathrm{ctms}$.

\section{Mortalidad Materia:}

Citamos unicamente aquellas muertes debidas a la intervencion, pues at que se han visto con escasa frecuencia en las operada no siempre son ac cables a ésta.

Tres casos se tienen que anotar: uno debido a la hemomagia y dos en que se diagnosticó shock obstétrico; da un porcentaje igual al $1.7 \%$ en 1 intervenciones.

Mopitalidad infantil:

Mortalidad en los niños nacidos en cesarea:

En 171 casos, 10 muertes, lo cual da un porcentaje de $5.8 \%$ sensiblemes igual a los citados por el Dr. Capasso en su reciente trabajo y al encontrado él de $5.72 \%(3-4)$.

Tenemos que lamentar el no poder presentar ningun dato sobre los teresantes aspectos que representan las complicaciones post-operatorias y imposibilidad absoluta de estudiar los índices de morbilidad en las ope das; tal vez con otro tipo de historia más completo se pueda adelantar u investigación más rigurosa y no desperdiciar esa fuente que es el servi médico del ICSS. 


\section{Comentario}

Ricsulta inter sante observar las incidencias de Cesáreas anotadas y com paradas con las publicadas en otros páses. Nuestros resultados son aceptable: co'ocandonos en in térmiro medio en lo general.

Tambien resultan aceptables los resultados comparativos de la Clínica Primero de Mavo con las oras instituciones de la Ciudad y en este caso. se deben tener en cuenta el tipo de pacientes que a ella concurren; no se les pucte considerar como absoluta clientela hospitalaria, pero tampo_o partizular, lo cual ustifica su colozacion en un punto intermedio. En esa forma no se puede ano ar que la incidencia de la cesirea en la Clínica Primero de Mayo se encuenira dentro de cifras, que en ningun ciso se pueden considerar como cxageradas y que in pretender abolir o desechar la intervención si se nota una marcada tendencia a limitarla a aquellos casos de indicacion muy precisa.

Es muy de lamentar la falu de las historias que no nos fue posible tomar on cuenta en este estudio: de estas la mavoría no se encontró en el archivo y una poca cantidad turo que ser desechada por carecer de valor cientifico. Lo mismo hay que decir de los cuadros de temperatura y hojas de donde quedan consigwdas las indicaciones méticas y evoluciones del post-operatorio, pues la ausen in total de éstas impide analizar la morbilidad y las diferentes complicaziones wcedidas después de la interrención.

Los porcentajes que indican la incidencia según la paridad, son sensible. mente iguales a los publicados por los Hospitales Americanos, coincidiendo en un mayor número en las multiparas.

\section{DESPROPORCIÓN CÉFALO-PÉLVICA}

Alobservar el resumen de indicaciones por las cuales se llevaron a cabo las mervenciones, aparecen en primer término 78 bajo el diagnóstico de Desprorcion Céfalo-Pélvica.

Este tema de por si serla suficiente para adelantar un trabajo de gran in : res, pues bien sabidas son las variadas circunstancias y condiciones bajo las cuales se plantea este diagnostico. Sin embargo cabe anotar que nuestras estaHinticas se dividen de la siguiente manera:

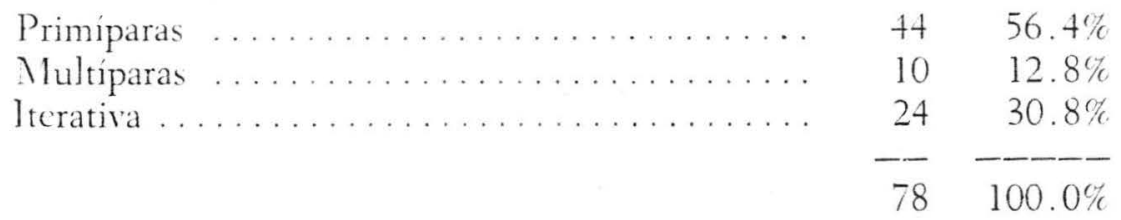

Todos los autores están de acuerdo en aceptar la mayor incidencia en las frimiparas; pero en el caso de multíparas es en donde el factor Feto desempeña un papel más importante, siendo innegable el mayor desarrollo de éste con relacion a la paridad en casos absolutamente normales. 
Pero antes de seguir adelante séanos permitido hacer un comentario m personal sobre las historias y el diagnóstico de "DESPROPORCION CEFAL PELVICA. En la iniciación de este trabajo anotamos la imposibilidad de come tar el $31.8 \%$ del total de las historias, e indicamos las razones para ello.

Para los que conocemos las capacidades y preparación de quienes han $\mathrm{tr}$ bajado con nosotros en la Clínica, no habría motivo alguno para dudar de las zones bajo las cuales se sustentaron estos diagnósticos; pero siendo los archivos d ICSS fácilmente consultables por quienes estén interesados en la obtención datos estadísticos y de valor médico, lógicamente discutirían este diagnóstico, incidencia y porcentaje; porque en 78 casos, 14 de ellos o sea el $19.2 \%$ carect del indispensable dato de pelvimetría externa.

En todos los casos se empleó en forma sistemática la prueba de trabajo y és fue adelantada con un criterio distinto según se tratara de primíparas, multípar o iterativa.

El promedio global fue de 18 horas.

En las primíparas el promedio de la prueba de trabajo fue de 16 horas: las multiparas la gran mayoría se aguardó a que la dilatación cervical fuera cor pleta $\mathrm{y}$ previa ruptura artificial de membranas se intento la aplicación de $\mathrm{fo}$ ceps. la que generalmente falló, obligando a intervenir por via alta.

Los casos de cesárea iterativa fueron sometidos también a la prueba, pe en estos casos no se desechó el peligro de la ruptura uterina y siendo en tod comprobada la desproporción, únicamente se permitio una duración de traba suficiente para lograr buena formación del segmento y una dilatación cervic que permitiera la fácil expulsión posterior de loquios.

\section{Placenta previa}

Continua en orden de frecuencia la placenta previa con 22 casos; consid ramos sin objeto insistir una vez más sobre la gravedad que encierra esta entidi tanto para la madre como para el feto. Las presentadas en este estudio se divid de la siguiente manera:

Placenta previa central total

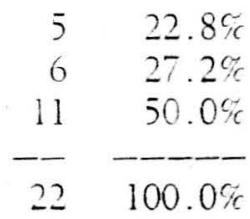

Las condiciones fetales fueron:

Fetos normales.

$13 \quad 59.1 \%$

" prematuros

mortinatos

,

Sin dato 


\section{Toxicosis GravíDica}

La incidencia de Toxicosis gravídica, es de 21 casos.

Se dividen en la siguiente forma:

Multíparas

$\begin{array}{rr}6 & 28.6 \% \\ 15 & 71.4 \% \\ -21 & 100.0 \% \\ 9 & 42.9 \% \\ 9 & 42.9 \% \\ 1 & 4.7 \% \\ 2 & 9.5 \% \\ -21 & -100.0 \%\end{array}$

La acción sobre el feto se manifestó asi:

Fetos a término

$$
\begin{array}{rr}
13 & 62.0 \% \\
8 & 38.0 \% \\
\hline 21 & 100.0 \% \\
1 & 4.7 \% \\
0 & 0.0 \%
\end{array}
$$$$
\text { Prematuros }
$$

Mortalidad materna

Mortalid dainfantil

El promedio de tensión arterial para estos casos fue: Mx. 150, Mn.96. Debc anotarse el hecho bien sabido de las condiciones de inferioridad en que nace el hijo de madre eclámptica y más si ésta tiene que ser intervenida, cuando el feto upenas alcanza los límites de la viabilidad. El hecho establecido en la Clínica de ue todo niño prematuro o a término nacido en condiciones pobres de salud, fuetrasladado a una clínica distinta y especializada. impidio a los obstetras el seir de cerca la evolución de esos niños; posiblemente al confrontar las estadícas de mortalidad infantil en la Clinica de Nutricion, se pueda encontrar ciI...s que no corresponden a las presentadas en este trabajo.

\section{Situación transversa}

La situacion transversa se presentó en 10 casos.

En 8 de éstos se encontró en el momento de ingreso a la Clínica, feto vivo y membranas rotas, lo cual justifico ampliamente la intervención. De los 2 resintes en uno no figura ningún dato que explique la conducta: el otro que nos có seguir de cerca, fue el de una multípara que ingresó a la Clínica con 15 hos de trabajo, dilatación de 5 ctms. y bolsa integra.

Al examen se encontró: Embarazo a término con feto vivo en situación transiersa A.I.D.P. Se intentó en dos ocasiones variar la situación fetal por medio de iersión externa, pero en ambas ocasiones se notó la desaparición de ruidos feta- 
les y el inmediato retorno a la situación primitiva; en vista de lo cual, discutia el caso en junta médica se resolvió intervenirlo por váa alta. Llevada a efec esia, se comprobó la presencia de cuatro circulares del co:dón alrededor del cu 1lo fetal. Como nos pareciera de interés el caso hicimos la mejizion de los cata encontrando una longitud de 23 ctms., para el cabo proximal y 36 para el dist

\section{Distocia cervical}

11 intervenciones fucron practicadas bajo este diagzosico

2 po: aglutinación del cuello.

1 por rigidez cicatricial.

En las 8 restentes no se -ncuentran datos que permitan clasificarlas.

\section{Primigestantes de edad avanzadi}

Cuatro primiges:antes, cuva edad promedio fue 35 aros. fueron intervenit por esta causa y después de haber sido todas sometidas a prueba de trabajo, $q$ en promedio duró 30 horas, no se logré el encajamiento, ni la dilatación cuello.

\section{Presentaciones distócicas}

Incluímos en este punto dos presentaciones de frente en multipàras, que gresaron con bolsa rota, trabajo prolongado y en las que la mutacion falló.

Una presentación de cara en variedad posterior en primigestante. con bo rota ! diametros pélvicos pequeños.

\section{Catisas varias}

Embarazo gemelar en primípara ................ I caso.

Esterilidad convugal de más de 8 años ............. I caso.

Endocarditis reumatoide ................. I caso.

Fistula rectovaginal operada $\ldots \ldots \ldots \ldots \ldots \ldots \ldots \ldots \ldots$ l caso.

\section{Mortalidad materna}

Las defunciones ocurridas fucron comentadas anteriormente.

\section{Mortalidad infantil}

Los porcentajes anotados corresponden únicamente a aquellas defunci infantiles que se sucedieron en la Clinica, antes de que los niños fueran tr dados a la Clínica de Nutrición. 


\section{CONCLUSIONES}

1. - Hasta el 31 de diciembre de 1951 fueron atendidos en la Clínica Primero de Mayo 7.909 nacimientos que dieron lugar a 251 intervenciones cesáreas, con un porcentaje del $3.17 \%$.

2. - El porcentaje de cesáreás en la Clínica Primero de Mayo es el más bajo entre las Instituciones locales comparadas.

3. - Se registra una disminución del $1 \%$ en la incidencia de cesáreas, que coincide con el establecimiento de juntas médicas reglamentarias.

4. -De las historias consultadas $31.8 \%$ no se puede utilizar.

5. - La mayor incidencia de cesáreas se registra en las multíparas.

6. - Las indizaciones que dieron lugar a un mayor número de intervenciones fueron: Desproporción Céfalo-Pélvica, Placenta previa, Eclampsia y Distocia cervical respectivamente.

7. - La edad comprendida dentro de los 20 y los 30 años es la más afectada por la cesárea.

8.--El peso promedio de niños nacidos por cesárea es de 2.985 gms., la talla promedio es de $48.5 \mathrm{ctms}$.

9. - La mortalidad materna corresponde al $1.7 \%$.

. - L La mortalidad infantil corresponde al $5.8 \%$.

- Las causas que motivaron las interrenciones se encuentran comprendidas dentro de las indicaciones de ésta.

2. - El tipo de historia que se emplea es poco útil para adelantar estudios médico-estadísticos.

13. - No existe intervencionismo quirúrgico en la Clínica Primero de Mayo.

\section{B I B L IO G R A F I A}

-An Analysis of Ten Years of Cesarean Section at the Cincinnati General Hospital. Robert W. Kistner M. D. Cincinnati, Ohio. A. J. of Obs. \& Gin. Enero 1951, Nơ, 1, Vol. 61, pág. 111.

2.-A Study of Cesarean Sections at Jefferson Davis Hospital. Melville L. Cody M D. Houston. Texas, A. J. of Obs. \& Gin. Agosto 1951. No 2, Vol. 62, pág. 415.

3.-. Consideraciones sobre 3.000 partos". Dr. Camilo Capasso. Rev. Col. de Obs. y Gin. Vol. No 3, 1952. pág. 113.

-An evaluation of Cesarean Section in Infant mortality. Carl P. Huber M. D. Indianapolis. Ind. A. J. of Obs \& Gin. Abril de 1951. Vol 61, No 4, pág. 897.

- Principles and Practice of Obstetrics. De Lee-Greenhill, 9th. Edition Saunders 1947. pág. 915.

6. - "Tratado de Obstetricia". S. Dexeus Font Salvat. Barcelona 1949. 